Cerebrovasc Dis 2007;24:144-146

DOI: $10.1159 / 000103619$

\section{Transoral Carotid Ultrasonography Is Useful for Detection and Follow-Up of Extracranial Internal Carotid Artery Dissecting Aneurysm}

\author{
Yusuke Yakushiji a, Yukinori Takase ${ }^{\mathrm{b}}$, Masafumi Kosugi ${ }^{\mathrm{a}}$,

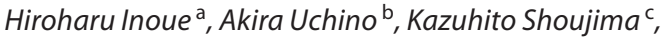 \\ Yasuo Kuroda ${ }^{\text {a }}$
}

aDivision of Neurology, Department of Internal Medicine, and

${ }^{b}$ Department of Radiology, Saga University Faculty of Medicine, and 'Shoujima Neurosurgery Hospital, Saga, Japan

The development of neuroimaging techniques has been improving the diagnosis of extracranial internal carotid artery dissection (EICAD) [1]. Recently, transoral carotid ultrasonography (TOCU) has also been shown as a useful noninvasive procedure for the diagnosis of EICAD [2-4]. We herein report that TOCU is quite useful for the detection and follow-up of EICAD complicated with aneurysm.

\section{Case Report}

A 62-year-old man having a history of lacunar infarction in the left putamen 4 years previously was referred to a regional hospital with severe headache of sudden onset (day 0). He happened to have had brain magnetic resonance angiography 10 months before, which showed no abnormalities. He had a history of no cardiovascular diseases, hypertension, hyperlipidemia and cervical and cranial trauma. There was no family history of cardiovascular diseases. He was almost normal on general physical and neurological examinations. Although diffusion-weighted magnetic resonance imaging showed no acute ischemic lesions in the brain, magnetic resonance angiography showed the complete disappearance of the right internal carotid artery (ICA). On carotid angiography (CAG), luminal irregularity, severe stenosis and aneurysmal form were found at the $\mathrm{C}_{1}$ vertebral level of the right ICA.

He was transferred to our hospital, and conventional carotid ultrasonography was performed on day 29 , which, however, did not find any difference in the diameter and the end-diastolic flow velocities between bilateral common carotid arteries (right 30.3 $\mathrm{cm} / \mathrm{s}$, left $31.7 \mathrm{~cm} / \mathrm{s}$ ). The right CAG performed on day 32 showed the persistence of luminal stenosis and aneurysm-like dilatation, with mild improvement of the stenosis (fig. 1a). On the same day, TOCU was first performed after informed consent. We used Sonosite Titan version C 2.2 (Sonosite Inc., Bothell, Wash., USA) equipped with an 8 - to $5-\mathrm{MHz}$ convex array transducer $(\mathrm{C} 8 / 8-5$ $\mathrm{MHz}$ ) [2]. The transducer was originally designed for prostate imaging and was equipped with a color power Doppler imaging system. The probe tip was painted with echo jelly, covered with a clean cover and softly attached to the posterolateral pharyngeal wall. The attachment of the probe tip to the pharyngeal wall was confirmed by roentgenograms (fig. 1b). TOCU clearly demonstrated the dilatation of the right ICA (diameters: right, $8.2 \mathrm{~mm}$; left, 4.0 $\mathrm{mm}$ ) with narrowing of the true lumen $(3.3 \mathrm{~mm}$; fig. 1c). The aneurysm sac observed on CAG was shown as a protrusion toward the ventral side (the whole length, $2.5 \mathrm{~mm}$; intimal tear length, 7.3 $\mathrm{mm})$. In the false lumen, the isoechoic area indicating intramural hematoma was present around the aneurysm sac, of which thickness was $2.6 \mathrm{~mm}$ from the brim of the aneurysm sac to the arterial adventitia. A severe stenotic flow pattern was observed at the distal part of the aneurysm sac (peak systolic flow velocity: right, $232.6 \mathrm{~cm} / \mathrm{s}$; left, $120 \mathrm{~cm} / \mathrm{s}$ ). The peak systolic flow velocity ratio (right ICA/ left ICA) was increased to 1.98. On magnetic resonance angiography performed on the same day, the size of the ICA was larger on the right and axial source imaging of time of flight depicted the double lumen in the right ICA: one lumen was hyperintense, indicating blood flow, whereas the other was isointense, indicating intramural hematoma in the false lumen (fig. 1d). The false lumen protruded toward the pharyngeal wall. All these findings suggested a diagnosis of spontaneous EICAD. He was treated with long-term antiplatelet (aspirin) therapy. TOCU was performed once a month thereafter, which confirmed the gradual improvement of the narrowing of the true lumen and the decrease in size of both intramural hematoma and aneurysm sac. The peak systolic flow velocity at the distal portion of the right ICA also decreased to that of the left ICA on day 118 (right $92.6 \mathrm{~cm} / \mathrm{s}$; left $97.4 \mathrm{~cm} / \mathrm{s}$; flow velocity ratio 0.95 ). On day 245 , the protrusion and intimal tear of the aneurysm sac were found to decrease to 1.7 and $5.8 \mathrm{~mm}$, respectively (fig.1e). Intramural hematoma, which had been seen at the ventral side of the affected ICA on the first TOCU, was seen at its dorsal side. At the follow-up after 10 months, the patient displayed neither ischemic events nor iatrogenic adverse effects, but mild headache still remained.

\section{Discussion}

EICAD preferentially occurs below the $\mathrm{C}_{1}-\mathrm{C}_{2}$ vertebral level [5]. The development of a dissecting aneurysm was reported in approximately $25-50 \%$ of EICAD [5-8], which was usually located at the subpetrous portion $[5,8]$, that is within the range of observation by TOCU [2]. TOCU was shown to be useful in the diagnosis of EICAD [2-4], but the success in detecting an aneurysmal form, which is one of the main angiographic findings of arterial dissection [9], by TOCU has never been reported in EICAD.

Arterial dissections causing intimal tears allow the penetration of blood into the arterial wall. The intramural hematoma often splits the media, often expands to the adventitia and creates a dissecting aneurysm. Thus, the wall of a dissecting aneurysm is composed of the media, the external elastic lamina and the adventitia [1]. The rupture of this dissecting aneurysm was reported to be rare in EICAD even when antithrombotic agents were being administered $[7,8]$. In our patient, TOCU revealed the dissecting aneurysm to be almost entirely covered with thick intramural hematoma, suggesting that the rarity of a rupture of a dissecting aneurysm is dependent on the thickness of the intramural hematoma, because it could keep the arterial adventitia from the direct exposure to blood pressure.

In conclusion, aneurysms on CAG would reflect only a defect of the intramural thrombus. We demonstrated here in a patient with EICAD that TOCU is able to detect all the false lumen, intramural hematoma and aneurysmal sac, indicating a most useful procedure for repetitive, economical, noninvasive observation of whether a dissecting aneurysm is in the impending state of rupture.

\section{Acknowledgement} tions. 

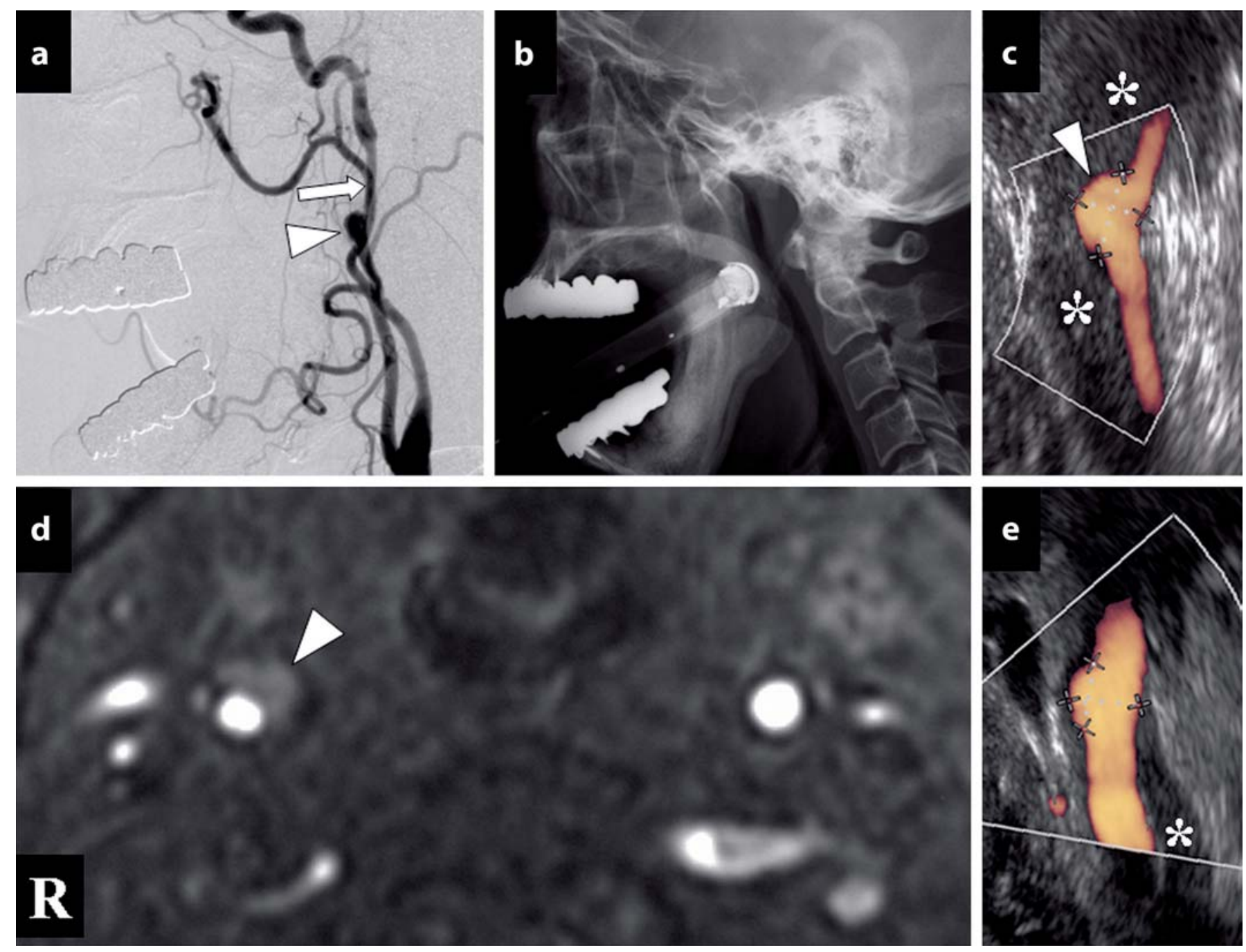

Fig. 1. a Lateral view of right common CAG, performed on day 32, shows luminal stenosis (arrow) and aneurysmal form (arrowhead) of the ICA at the $\mathrm{C}_{1}$ vertebral level. b The skull roentgenogram confirms the attachment of the probe tip to the pharyngeal wall. c Color power Doppler TOCU demonstrated the dilatation of the right ICA with narrowing of the true lumen. Intramural hematoma (asterisks) is also seen around the aneurysmal sac (arrowhead). d Axial source image of time of flight depicts the double lumen of the right ICA: one lumen shows hyperintensity, indicating blood flow, whereas the other exhibits isointensity, indicating intramural hematoma (arrowhead). e Follow-up TOCU on day 245 reveals a decrease in the dissecting aneurysm. Intramural hematoma is seen at the dorsal position of the affected ICA (asterisk).

\section{References}

1 Mokri B: Cervicocephalic arterial dissections; in Bogousslavsky J, Caplan L (eds): Uncommon Causes of Stroke. Cambridge, Cambridge University Press, 2001, pp 211-229.

2 Yasaka M, Kimura K, Otsubo R, Isa K, Wada K, Nagatsuka K, Minematsu K, Yamaguchi T: Transoral carotid ultrasonography. Stroke 1998;29:1383-1388.

3 Koga M, Kimura K, Minematsu K, Yasaka M, Isa K, Yamaguchi T: Transoral carotid ultrasonographic findings in internal carotid artery dissection - A case report. Angiology 2000;51:699-703.

4 Yakushiji Y, Yasaka M, Takada T, Minematsu K: Serial transoral carotid ultrasonographic findings in extracranial internal carotid artery dissection. J Ultrasound Med 2005;24:877-880.

5 Mokri B, Sundt TM Jr, Houser OW, Piepgras DG: Spontaneous dissection of the cervical internal carotid artery. Ann Neurol 1986;19:126138.

6 Biousse V, D’Anglejan-Chatillon J, Touboul PJ, Amarenco P, Bousser MG: Time course of symptoms in extracranial carotid artery dissections: a series of 80 patients. Stroke 1995;26:235-239. 
7 Guillon B, Brunereau L, Biousse V, Djouhri H, Levy C, Bousser MG: Long-term follow-up of aneurysms developed during extracranial internal carotid artery dissection. Neurology 1999;53:117-122.

8 Touze E, Randoux B, Meary E, Arquizan C, Meder JF, Mas JL: Aneurysmal forms of cervical artery dissection: associated factors and outcome. Stroke 2001;32:418-423.

9 Hart RG, Easton JD: Dissections of cervical and cerebral arteries. Neurol Clin 1983;1:155-182.

\section{Dr. Yusuke Yakushiji}

Division of Neurology, Department of Internal Medicine

Saga University Faculty of Medicine, 5-1-1 Nabeshima

Saga 849-8501 (Japan)

Tel. +81952 34 2360, Fax +81952342017

E-Mail yakushij@cc.saga-u.ac.jp

Cerebrovasc Dis 2007;24:146-148

DOI: $10.1159 / 000103620$

\section{Takotsubo Cardiomyopathy - A Rare Cause of Cardioembolic Stroke}

André Grabowski a Juergen Kilian ${ }^{\text {b }}$, Cornelia Strank ${ }^{a}$, Gerhard Cieslinski ${ }^{\text {b }}$ Uta Meyding-Lamadéa

Departments of a Neurology and ${ }^{\mathrm{b}}$ Cardiology, Krankenhaus

Nordwest, Frankfurt/Main, Germany

Apart from the classic macro- and microangiopathic vascular changes, cardiac embolism represents an important factor in the etiology of stroke. Most people suffering a cardioembolic stroke have atrial fibrillation as the major cause, but cardiac dysfunction is also a potential risk factor for embolic ischemia [1].

\section{Case Report}

We present 2 female patients who were admitted to our stroke unit due to acute neurological deficits. The first patient was an 85-year-old woman who developed an acute palsy of her right arm. Cranial MRI revealed multiple ischemic lesions in both hemispheres (fig. 1).

The 64-year-old second patient presented with a Broca aphasia on admission. In the cranial CT scan a middle cerebral artery territorial infarction of the left hemisphere was seen.

Both women also had signs of an acute coronary syndrome on admission, with an ST segment depression and T wave negativity of the anterior wall in the ECG and moderately elevated troponin levels in the blood assay (3.31 and $3.70 \mathrm{ng} / \mathrm{ml}$ ) but without typical clinical symptoms like chest pain or dyspnea.

In both patients, a coronary angiography showed normal arteries without any signs of stenosis or sclerosis. In the ventriculography, a midventricular and apical hypokinesia was present, appearing like an 'apical ballooning' (fig. 1,2). The left ventricular function (LVF or ejection fraction, EF), measured by echocardiography and ventriculography, was reduced to 30 and $45 \%$, respectively. Patient 2 additionally had an apical thrombus in the left ventricle (fig. 2), therefore an anticoagulation therapy with warfarin was started to prevent further embolic lesions.
Due to the findings in the coronary angiography a relevant coronary heart disease was excluded. In further investigations other causes of a stroke (e.g. stenosis of the brain-supplying arteries, arrhythmias or atrial fibrillation) were not identified.

Within the following days the troponin levels decreased to a normal level.

The control echocardiography performed a few weeks later showed normalized cardiac function in both patients.

The diagnostic findings and the course of this syndrome suggest that both patients had an embolic ischemic stroke due to a tako tsubo (or stress) cardiomyopathy.

\section{Discussion}

Takotsubo cardiomyopathy or 'stress cardiomyopathy' is a rare syndrome, which was first described in the early 1990s in Japan [2]. Its Japanese name is derived from the antique octopus trap (takotsubo), which is shaped like an ampulla. Within the last few years, several case reports and few small case series were published with a total number of less than 400 patients [3-17]. In most cases takotsubo cardiomyopathy affected elderly women with previous emotional, psychological or physical stress. It often looks like an acute coronary syndrome with chest pain, dyspnea, ECG changes, elevated troponin and a left ventricular dysfunction [18] (EF often less than 50\% [10, 16, 19]), which is seen as the typical 'apical ballooning' in the ventriculography, but without any signs of coronary heart disease in the coronary angiography. The left ventricular dysfunction in this syndrome is a reversible phenomenon and the prognosis was generally excellent [18]. However, the cardiac dysfunction itself as well as ensuing complications like the formation of an intraventricular thrombus, as seen in the second patient of our report, can lead to a cardioembolic stroke. So far, only 4 case reports can be found in the literature describing ventricular thrombus in apical ballooning [20, 21]. In 2 of these cases patients suffered a cerebral ischemia $[22,23]$. In one case series 3 patients with stress cardiomyopathy had a previously described 'cerebrovascular accident' [24].

In our case report the conclusion that the cerebral ischemia was the result of the left ventricular dysfunction with cardiac embolism seemed to be very likely due to the exclusion of other causes of cardiac embolism. But on the other hand one has to consider other explanations, e.g. that takotsubo cardiomyopathy might be the consequence of 'stroke-related stress'. This stress-related phenomenon was seen in our hospital in some patients after having an operation. These patients had a normal ECG before the operation, and in their further course they showed ECG changes and an apical ballooning. In our opinion the clinical course is an important factor in the etiological considerations, whereas stress factors (e.g. operations or acute onset of diseases) might implicate that apical ballooning is secondary.

Another more hypothetical explanation might be that the cerebral ischemia itself may cause a 'neurocardiac-injury syndrome' as seen in some patients with subarachnoid hemorrhage $[25,26]$. In these cases a stress-related phenomenon is possible as well [27]. Since large series of stroke patients with stress-induced cardiomyopathy do not exist, the 'chicken or egg' question is not easy to answer and further research, especially epidemiological data, is necessary. In view of the increasing number of stroke patients every year, takotsubo cardiomyopathy cer- 